\title{
New insights into the structural and dynamics properties of collagen model peptides using CD spectroscopy, MD simulations and innovative NMR approaches
}

\author{
Emeric Miclet ${ }^{1}$, Anas Terrien ${ }^{1}$, Claire Loison ${ }^{2}$, Luminita Duma ${ }^{3}$, Thierry Brigaud ${ }^{4}$, Nathalie Lensen ${ }^{4}$ \\ Keyvan Rahgoshay ${ }^{4}$, Grégory Chaume ${ }^{4}$ \\ ${ }^{1}$ Sorbonne Université, France \\ ${ }^{2}$ Université Lyon 1, France \\ ${ }^{3}$ UTC, France \\ ${ }^{4}$ Université de Cergy Pontoise, France
}

https://doi.org/10.17952/35EPS.2018.192

Collagen, the most abundant protein in the animal kingdom, is also an important biomaterial owing to its great mechanical strength and its excellent thermal stability. This last decade, both the industry and the academia have been involved in the development of new collagen-related biomaterials.[1] One common strategy consists in using Collagen Model Peptides (CMP) as collagen surrogates. Numerous studies have demonstrated that short sequences, based on the repetition of the Pro-Hyp-Gly triplet, can be used to mimic the primary sequence of collagen.[2]

Using such peptides, we have developed an approach combining CD and NMR spectroscopies in order to characterize the formation of the triple helix (TH) and its structural features in solution. MD simulations and NMR spectroscopy have allowed us to gain insights into the conformational ensembles explored by the monomeric CMP peptides and their conversion into larger oligomeric species. Innovative $13 \mathrm{C}, 15 \mathrm{~N}$ strategies and NMR developments have been carried out to obtain structural information on these assemblies at the atomic scale. In addition, we have been able to introduce 19F nuclei in model CMP using previously reported strategies.[3-6] Kinetic and structural effects of this insertion onto the collagen triple helix have been discussed using the developed biophysical tools and MD simulations.

\section{Structural requirements for the stability of the collagen Triple Helix}

The primary structure of collagen has a high content of (Pro-Hyp-Gly) units. The secondary structure adopts a PPII conformation: all peptide bonds are trans, $\alpha$ and $\psi$ dihedral angles are $-70^{\circ}$ and $150^{\circ}$, respectively. The stability of the right-handed triple helix (TH) is ensured by inter-strand hydrogen bonds and the downup alternation in puckering of pyrrolidine rings (figure 1). [2] Glycine residues are regularly distributed along the peptide sequence and are thus valuable probes of the TH structure.
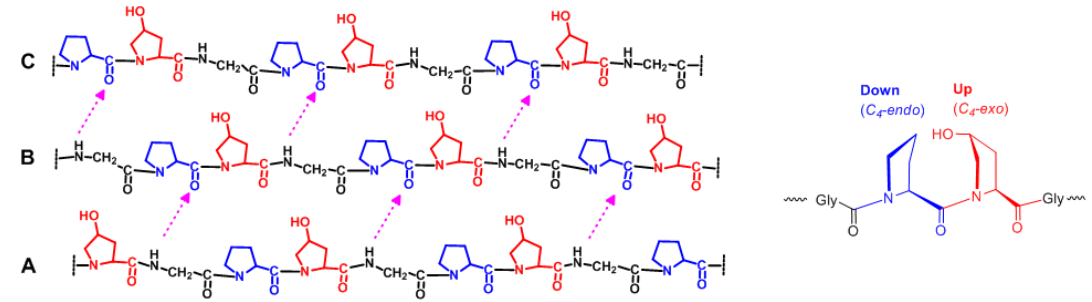

Figure 1: Hydrogen bond pattern between chains A, B and C in the collagen TH (left) and puckering alternation in Pro-Hyp pairs

\section{Use of the CH2-TROSY NMR spectroscopy to observe methylene groups in TH models}

The CH2-TROSY pulse scheme is based on the Rance-Kay ${ }^{1} \mathrm{H}_{-}{ }^{13} \mathrm{C}$ HSQC experiment. A spin state selective element has been introduced prior to the $t_{1}$ evolution making it possible to select the ${ }^{13} \mathrm{C}$ downfield component. Then, by removing both the ${ }^{1} \mathrm{H}$ decoupling during the ${ }^{13} \mathrm{C}$ evolution and the ${ }^{13} \mathrm{C}$ decoupling during the acquisition and by adjusting the transfer delays in the double INEPT, it is possible to suppress the ${ }^{2} \mathrm{~J}_{\mathrm{HH}}$ and to accurately measure small ${ }^{3} \mathrm{HH}$ couplings. This strategy was applied on specifically ${ }^{13} \mathrm{C},{ }^{15} \mathrm{~N}$ Gly-labelled CMPs and allowed the measurements of ${ }^{3} \mathrm{~J} / \mathrm{HN}$ along the peptide sequence (figure 2). Several conformers of the (ProHyp-Gly $)_{7}$ peptide were characterized using this approach and the ${ }^{3} \mathrm{~J}_{\mathrm{H} \alpha \mathrm{HN}}$ values measured at each Gly position are used to constrain the $\alpha$ angles in MD simulations. 

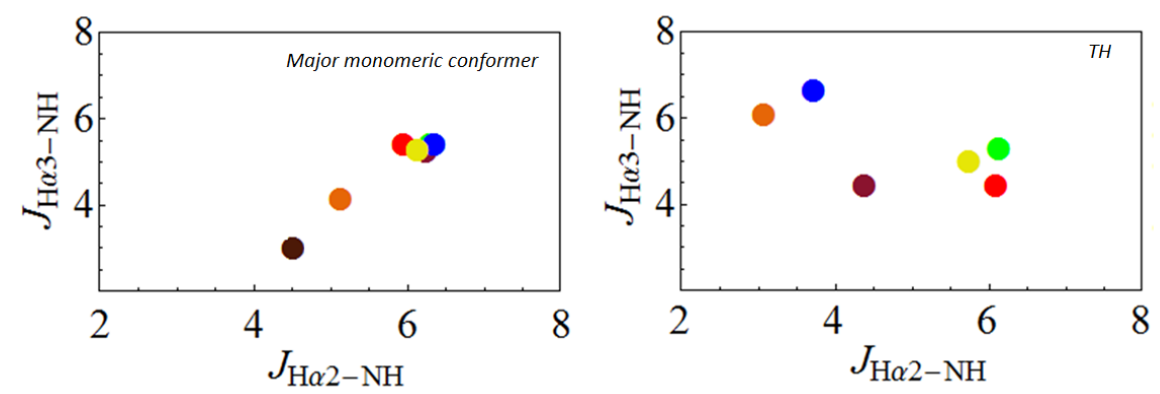

Figure 2: 3JHaHN couplings measured along the (Pro-Hyp-Gly)7 peptide sequence using the CH2-TROSY-pulse scheme. The two diastereotopic protons $\mathrm{Ha} 2$ and $\mathrm{Ha} 3$ of the Gly residues provide a measurement of the f-dihedral angle for each (Pro-Hyp-Gly)7 conformer.

\section{TH formation in fluorinated CMP}

FluorinatedCMPs have been synthetized by replacing Pro and/orHyp residues by $\mathrm{CF}_{3}$-substituted pseudoprolines.[3-6] The stability of the TH on these new CMP was monitored by both CD spectra and NMR spectroscopy (figure 3). In CD spectra, the dependence of the ellipticity at $225 \mathrm{~nm}$ as a function of the temperature gave an estimation of the TH melting temperature (Tm) since sigmoidal curves were obtained and the Tm value corresponds to the inflexion point. By NMR, the integration of a $\mathrm{H} \delta$ Pro which belongs to the $\mathrm{TH}$ provided independent measurements of the Tm values. In addition, the quantitative analysis of the 2D NMR spectra allowed us to evaluate at each temperature the population of each CMP conformers (TH and up to six monomeric states).
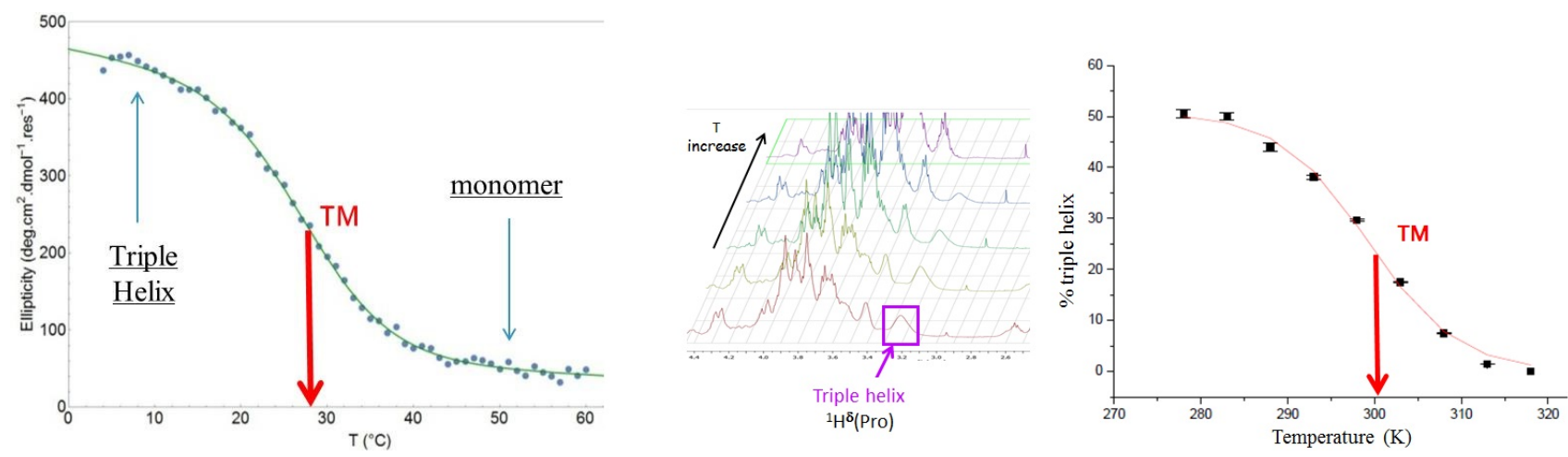

Figure 3: Monitoring the TH as a function of the temperature. $\mathrm{CD}$ spectra were recorded between $4{ }^{\circ} \mathrm{C}$ and $60^{\circ} \mathrm{C}$ and the [q]225 values were plotted as a function of the temperature (left panel). 1 H NMR spectra obtained with increasing temperatures (middle). Integration of the Hd signal of the Pro residues within the TH as a function of the temperature.

${ }^{19} \mathrm{~F}$ NMR DOSY were recorded to show evidence of the TH formation in Fluorinated CMPs. A diffusion coefficient ratio of 1.58 was found between the monomer and the triple helix. MD simulations have been performed to gain insights into the structural features of these new $\mathrm{TH}$. It was found that the $\mathrm{C}^{\delta}$ atoms in $\mathrm{CF}_{3^{-}}$ pseudoprolines must have specific configurations to engender the required ring puckerings and that the puckering alternation was essential to stabilize the fluorinated $\mathrm{TH}$.

In summary, the results obtained showed evidence that $\mathrm{CF}_{3}$-substituted pseudoprolines can be introduced in collagen model THs although slightly less stable than their (Pro-Hyp-Gly) ${ }_{n}$ homologues. It was found that the alternation in puckering of the 5-membered cyclic residues was a key point to promote the $\mathrm{TH}$ formation. 


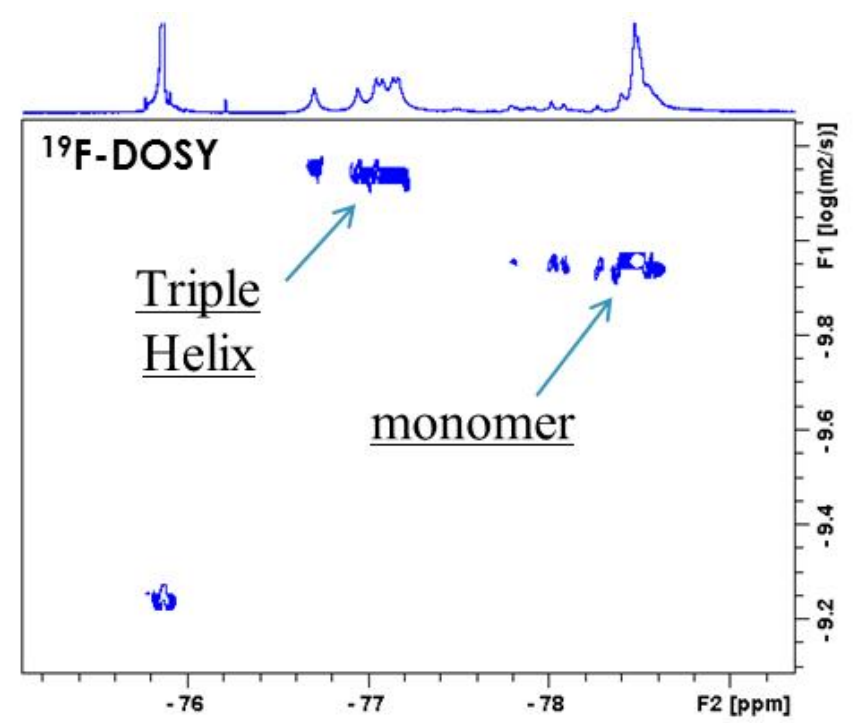

Figure 4: $19 F$ NMR DOSY spectra recorded on a Fluorinated CMPs.

\section{References}

[1] Koide, T., Philos. Trans. R. Soc. B. 362, 1281-1291 (2007).

[2] Shoulders, M. D., Raines, R. T., Annu. Rev. Biochem. 78, 929-958 (2009).

[3] Feytens, D., Chaume, G., Chassaing, G., Lavielle, S., Brigaud, T., Byun, B. J., Kang, Y. K., Miclet, E. J. Phys. Chem. B 116, 4069-4079 (2012).

[4] Chaume, G., Simon, J., Caupene, C., Lensen, N., Miclet, E., Brigaud, T., J. Org. Chem. 78, 10144-10153 (2013).

[5] Chaume, G., Feytens, D., Chassaing, G., Lavielle, S., Brigaud, T., Miclet, E. New J. Chem. 37, 1336-38 (2013).

[6] Chaume, G., Simon, J., Lensen, N., Pytkowicz, J., Brigaud, T., Miclet, E. J. Org. Chem. 24, 13602-13608 (2017).

[7] Guichard G., Violette A., Chassaing G., Miclet E. Magn. Reson. Chem., 46, 918-24 (2008). 